\title{
Diseño de un Modelo 3D del Politécnico Colombiano Jaime Isaza Cadavid con Realidad Virtual
}

\author{
Sandra P. Mateus y Jorge E. Giraldo \\ Politécnico Colombiano JIC, Facultad de Ingenierías, Cra 48 N 7-151 Of: P19-146, \\ Medellín-Colombia. (e-mail: spmateus@elpoli.edu.co; jegiraldo@elpoli.edu.co).
}

Recibido Ago. 09, 2011; Aceptado Oct. 04, 2011; Versión final recibida Dic. 05, 2011

\begin{abstract}
Resumen
Este trabajo muestra el desarrollo de un Entorno Virtual de la Sede Poblado del Politécnico Colombiano Jaime Isaza Cadavid, utilizando técnicas de realidad virtual. Esto con el fin de obtener un modelo de la planta física de la Institución que proyecte su imagen, a través de una interfaz gráfica amigable basada en tecnología 3D. El trabajo fue realizado en las siguientes etapas: i) caracterización de la planta física y diseño del modelo virtual, par lo que se seleccionaron 3DMax y Maya de Autodesk entre varias herramientas de modelado 3D y Unity como motor gráfico; ii) creación de mapas UV; y iii) proceso de texturización. Los resultados del entorno virtual desarrollado permiten al usuario navegar por la institución e interactuar con los diferentes espacios como si estuviera en un videojuego.
\end{abstract}

\section{Design of a 3D Model with Virtual Reality of the Colombian Polytechnic Institute Jaime Isaza Cadavid}

\begin{abstract}
This work shows the development of a Virtual Environment of the campus Poblado of the Colombian Polytechnic Jaime Isaza Cadavid, using virtual reality techniques. This with the aim of obtaining a model of the physical plant of the institution to project its image through a friendly graphical interface based on 3D technology. The work was developed following three main steps: i) characterization of the physical and virtual design of the model, for which 3DMax and Maya of Autodesk were selected among the several tools for 3D modeling and Unity as graphics engine; ii) creation of UV mapping; and iii) texturing process. The results of the proposed virtual environment allow the user to navigate around the institution and interact with the different spaces as done with a video-game.
\end{abstract}




\section{INTRODUCCIÓN}

Actualmente, el constante cambio en el mundo tecnológico y la creciente necesidad de nuevas y mejores tecnologías que emergen estocásticamente, ocasiona que muchos usuarios algunas veces se mantengan al margen de las nuevas herramientas que surgen para satisfacer demandas de visualización e interacción de modelos que manejan grandes cantidades de información. Así, con la aparición de estas nuevas tecnologías emergentes, una gran cantidad de conocimiento se ha generado y nuevas formas de llevar a cabo actividades y funciones se ven alteradas. Sin duda, el gran crecimiento en el desempeño de supercomputadoras y en particular de computadoras con grandes alcances en capacidades de graficación, ha permitido la incorporación de nuevas tecnologías de visualización y modelación como lo es la Realidad Virtual, la cual ha proporcionado un mejor entendimiento de un fenómeno o hecho real mediante su simulación tridimensional e interacción con equipo especializado.

En un Entorno Virtual, un computador genera impresiones sensoriales que son enviadas al usuario a través de sus sentidos, el tipo y la calidad de estas impresiones determinan el nivel de inmersión y la sensación de presencia en el Entorno Virtual. Lo ideal sería enviar al usuario información a todos sus sentidos, usando una alta resolución, alta calidad y consistencia en todo momento. Sin embargo, la realidad es muy diferente; muchas aplicaciones estimulan sólo uno o unos pocos sentidos, y muy a menudo utilizan una baja calidad en las representaciones y poseen una falta de sincronización de la información en el entorno virtual.

En cuanto a Entornos Virtuales, basados en Realidad Virtual, se pueden distinguir los siguientes trabajos: Ramírez et al. (1998), presentan el desarrollo de una aplicación hipermedial para la educación de museos haciendo uso del método OOHDM y las tecnologías subyacentes de la web. Monedero (2001) proporciona instrumentos de análisis a los arquitectos, de tal modo que puedan valorar más adecuadamente las consecuencias de una determinada intervención urbana tanto desde el punto de vista visual como desde otros puntos de vista, en la medida en que los modelos geométricos están asociados a datos alfanuméricos. Matteo et al. (2001), presentan el desarrollo de un Sistema de Realidad Virtual Inmersiva: VWC (Virtual World Creator), que contempla el diseño y creación de una herramienta de software que provee una interfaz que permite la construcción y edición de mundos virtuales inmersivos. Mármol et al. (2008), presentan la creación de una aplicación 3D interactiva, que genera recorridos virtuales, visualización de íconos históricos de varios puntos turísticos de la ciudad de Guayaquil. Quintero (2008), presenta el diseño y la construcción de un prototipo de bajo costo de un sistema de inmersión virtual de dos pantallas.

Banstalar et al. (2008) desarrollan un sistema de paseo virtual basado en web utilizando tecnología GIS, pero enfocado a sitios arqueológicos. Sheng et al. (2008) desarrollan un tour virtual 3D el cual soporta localización RFID aplicado al Museo Mackay. Xu et al. (2009) construyen un tour virtual basado en Google Earth, en el cual tienen inconvenientes con el volumen de información manejado. Shukol et al. (2011) proponen un método para reconstruir superficies planares de un ambiente real interno con oclusión y desordenado, resultando de ello un modelo 3D, pero este artículo está orientado más hacia la obtención de las escenas con laser. Wang et al. (2011) presentan la construcción de centros de clientes 3D virtuales, en el que se puede tomar un tour a través de internet. Sun et al. (2011) muestran un tour virtual 3D y basado en la web aplicado al Templo Tainan Confucian y direccionado a lo educativo. Carpenter et al. (2011) presentan un tour virtual de la Capilla Sixtina, presentando información de la Iglesia e inclusive citas bíblicas. Belloti et al. (2011) desarrollan una aproximación arquitectónica para la construcción de modelos urbanos, basados en mapas 2D y aplicando semántica.

En Colombia, existen pocas Universidades que contengan su Campus Virtual basado en Realidad Virtual, como es el caso de Rodríguez (2007) el cual plantea el desarrollo de un prototipo tridimensional para simular un recorrido virtual por las instalaciones de la Universidad El Bosque,; y el de Valencia y Vargas (2007) los cuales desarrollaron una aplicación 3D interactiva cuyo escenario es la Universidad Tecnológica de Pereira. Por el contrario, algunos denominados 
Campus Virtuales, son más bien páginas web estándar con animaciones flash, o por defecto simplemente fotografías del Campo de la Universidad, tal como es el caso actual del Politécnico.

Con base en lo anterior, este trabajo se centra en el desarrollo de Entorno Virtual 3D del Politécnico JIC, mostrando el diseño en detalle del Entorno Virtual, lo cual no se hace en otros artículos, el cual sumerja al usuario en un mundo virtual que recrea la realidad de los lugares de la Institución. En la siguiente sección se muestra el desarrollo del Entorno Virtual y posteriormente se exponen las conclusiones y trabajos futuros.

\section{DESARROLLO DEL ENTORNO VIRTUAL}

Con base en la información obtenida por medio de la observación, del registro gráfico de la infraestructura y del análisis de los datos obtenidos, se realizó la caracterización de la Planta Física de la Sede Poblado del Politécnico, etapa en la cual, se hicieron algunos planos de la Institución en una herramienta llamada Sweet Home 3D, con base en una estandarización.

En los inicios del proyecto se planteó realizar el recorrido por esquemas de sitio (stages), estableciendo que el recorrido se llevara a cabo por estaciones, las cuales indicarían los lugares más representativos del Politécnico. El recorrido se planteó de esta manera enfocado en la realización del recorrido en VRML. Posteriormente se decidió trabajar el modelado completo, para hacer el recorrido aún más real, y darle la posibilidad al usuario de realizar un recorrido total y de este modo reconocer todos los espacios de la Institución.

A lo largo del proyecto, se pusieron en consideración diferentes perspectivas de trabajo, entre ellos buscar la forma más adecuada en los aspectos que garantizan un óptimo desempeño en el ambiente 3D; así, se llegó a descubrir herramientas como Sketchup que es un programa computacional de modelaje y diseño en 3D, de fácil aprendizaje y grandes resultados en los proyectos realizados, pero tiene múltiples desventajas en consideración con las herramientas de Autodesk tales como motor de renderizado, apuesta a la geometría avanzada, manejo adecuado de texturas, control de polígonos, manipulación de efectos, y diferentes vistas en el momento del modelado. Posterior a esto, se tuvo en consideración la utilización de VRML como una herramienta de interacción con el ambiente, ya que esta representa modelos 3D, y como una de sus grandes ventajas es que puede ser exportado a la web, pero el principal problema de VRML es la interactividad, solo se puede realizar pocas operaciones, y además es limitado a tamaño de archivo, por otra parte Unity es un motor gráfico que soluciona muchos de los inconvenientes que representó VRML, ya que además de ser considerado uno de los mejores motores gráficos del mundo, sus costos no son elevados como lo llegan a ser muchos de los otros motores gráficos con igualdad de capacidades. Por las anteriores razones, las herramientas finalmente utilizadas fueron 3DMax y Maya de Autodesk y Unity.

Antes del modelado se acordó que el sistema de medidas fuese en unidades 3DMax y Maya a una escala de 1 unidad por metro (1:1) y que todas las rectas normales debían apuntar hacia la cara visible de la geometría, porque al momento de renderizado, solo estas caras con las rectas normales son mostradas, las que no, desaparecen y se ven espacios vacíos, además que no acepta ser texturizada.

También se tuvo en consideración de que debía tratar de reducir la cantidad de polígonos al máximo para hacer la malla de modelado más liviana y evitar desorden en las coordenadas y minimizar el tiempo de carga en la web y utilizar figuras planas en la medida de lo posible. De acuerdo a estas medidas de estandarización planteadas, se procedió a hacer todo el modelo del Politécnico (Ver Fig. 1). 

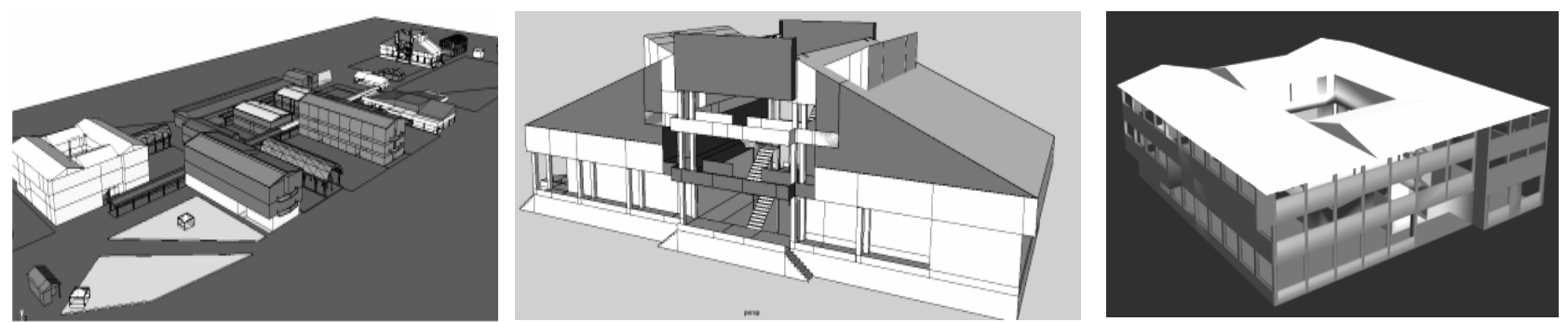

Fig. 1. Modelado del Politécnico

\section{Creación de Mapas UV}

Los mapas UV representan la forma de las figuras 3D en imágenes coplanarias, las cuales permiten su edición para darle color, es decir, darle textura. Para este trabajo de creación de mapas UV se tuvieron en cuenta dos procesos:

Proceso 1 - Limpieza de Geometría y Unión de Vértices: En el proceso de creación de los espacios del Politécnico, se le dio prioridad en principio y diseño a cumplir con la regla básica de geometría en la que cada plano tiene que contener solo 4 vértices, y al haberse basado todo el diseño en planos formando los espacios, esto era prioridad. Pero, al finalizar todo el proceso de diseño total del Politécnico, se encontró que se debía rediseñar cada vértice de los espacios, ya que muchos estaban siendo repetidos y desunidos en lugares que debían contener un solo vértice. En la mayoría de los casos, el proceso no es tan sencillo, algunos vértices no se dejan unir con otros, o terminan destruyendo la geometría y la posición y forma de los planos y bordes que los componen.

Todo el proceso anterior, consistió en ir juntando vértice a vértice, sin dañarles la posición inicial y la forma de la geometría, recordando que cada plano debe tener solo 4 vértices y probando que vértice puede unirse a otro. Cuando ningún vértice se deja unir, el proceso a seguir es rediseñar los planos que rodean este vértice evitando la sobrepoblación de vértices en un mismo punto. Así, se realiza la limpieza de geometría, por cada punto de convergencia de vértices se debían realizar estos procesos.

Proceso 2 - Creación de UV mapas: Este proceso consiste en crear el mapa basado en la dirección de las rectas normales con respecto al objeto y sus caras (Ver Fig. 2).

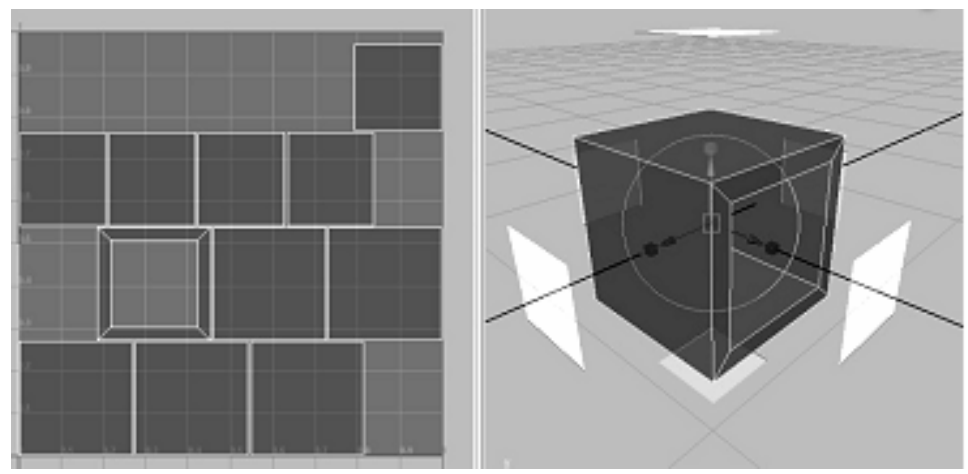

Fig. 2. Creación de Mapas UV

Después de lo anterior, se deben juntar las caras por sus uniones más significativas visualmente, es decir, que al ver el objeto en su estado final la textura se vea continua; este proceso se logra soldando los bordes comunes con la herramienta. Dado lo anterior, se debe llegar finalmente a algo parecido a lo que está en la parte izquierda de la Figura 2. Esto hay que hacerlo con cada objeto del modelado, y lograr darle continuidad en sus texturas para más realismo.

Una vez se terminan los mapas, se editan en algún programa de edición de imágenes, esto para poner los diferentes colores, materiales, texturas, rugosidad, etc.; de cada objeto para posteriormente importarlo en 3DMax o Maya programas en los que fue realizados inicialmente. En la Figura 3 se puede observar la transformación de una imagen de UV mapa y después la misma 
con su textura. En la parte derecha de la Fig. 3, se puede observar cómo queda el objeto con textura después de estos procesos.
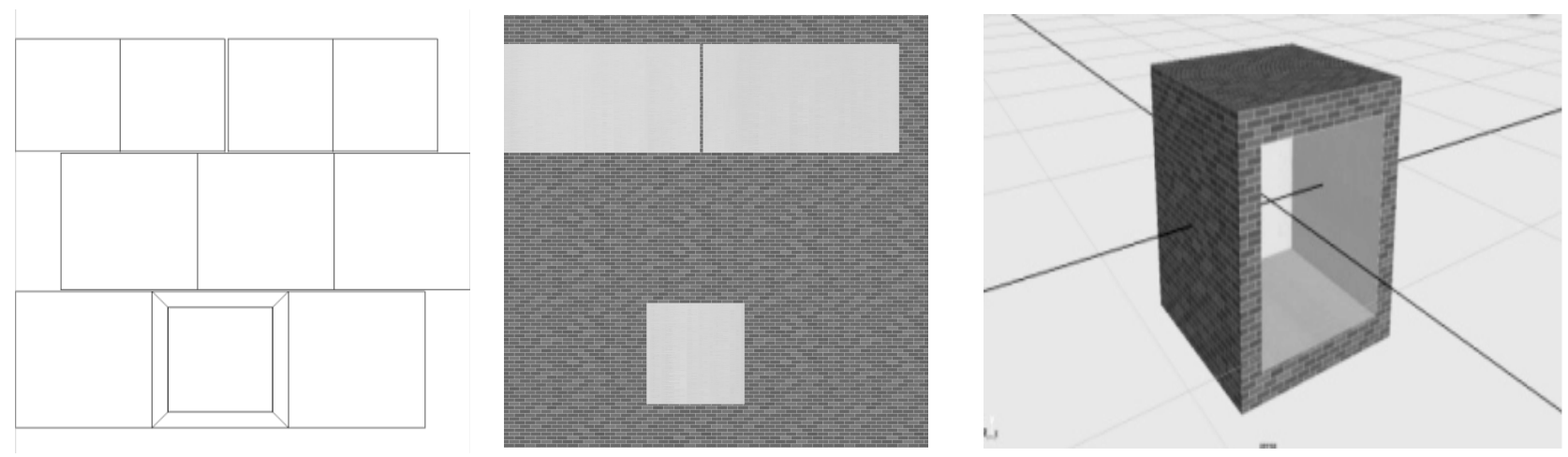

Fig. 3. Transformación de un Mapa UV a un Mapa con Textura

\section{Macro Modelo y Referencias}

Durante el proceso de modelamiento del Politécnico se encontró que trabajarlo modularmente facilitaría mucho la manera de edición y corrección de modelos y texturas, para lograr esto se utilizarían "referencias" que es una función de las herramientas de modelamiento 3D la cual consiste en cargar la geometría de otro archivo poniéndola en memoria y no en el archivo que hizo la referencia, así se logra que el macro modelo tenga una estructura de módulos. En algunas ocasiones la corrección o adaptación de los bloques fue necesaria por lo que se hacía más fácil trabajar sobre el módulo y no sobre el macro modelo.

El macro modelo es un archivo en el cual se verá todo el modelo del Politécnico, estará compuesto por modelos referenciados de los bloques, los cuales se encuentran en archivos individuales. Cada bloque del politécnico fue modelado en un archivo individual, de esta manera se pueden controlar los detalles de cada bloque por separado permitiendo que el trabajo sobre cada uno de ellos sea más rápido para el modelador, el texturizador y el computador. Teniendo modelos individuales de los bloques es necesario crear una colección de recursos unificados, para evitar diferencias inapropiadas entre los mismos, por ejemplo, si en varios bloques se usa la misma textura de ladrillos, las características deben ser definidas para que todos los bloques en los que se use luzcan igual, así se evitan incoherencias visuales en el macro modelo, de esto sale otra ventaja, la cual es poder manejar una colección simple de recursos independiente del motor en el que se tenga el modelo. Estando en el archivo principal (macro modelo) se crean referencia de los archivos de los bloques individuales, una referencia no es más que el archivo original dentro del principal (Ver Fig. 4).
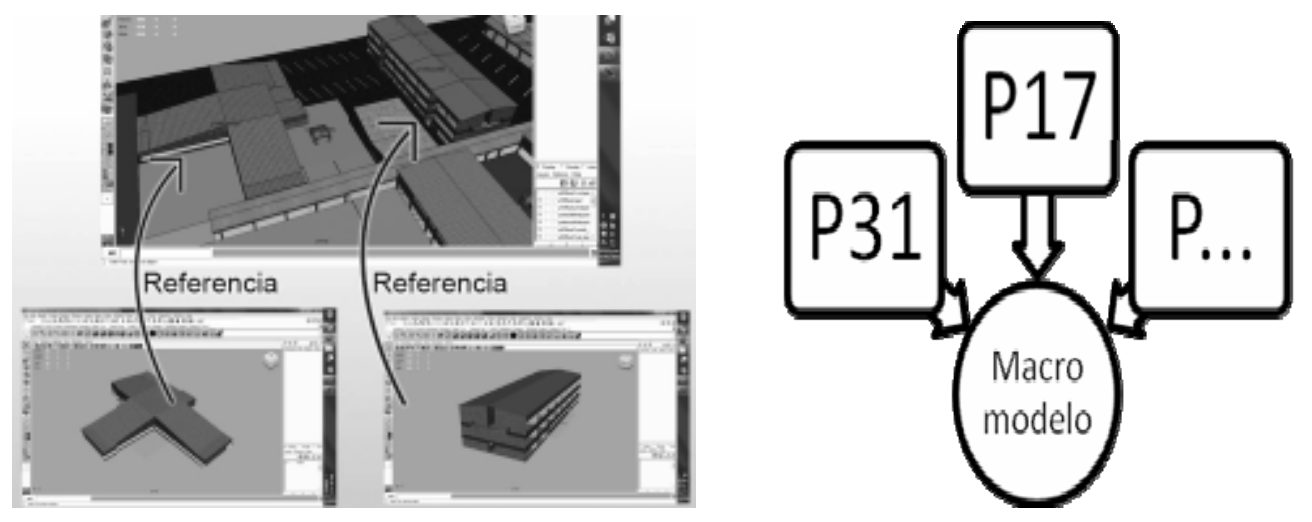

Fig. 4. Macromodelo y Referencias

\section{Mapas de Normales}

Uno de los últimos avances de la tecnología de motores gráficos es el mapa de normales (normal mapping), el cual consiste en una imagen de pixeles que al ser descompuestos en los colores 
principales rojo, azul y verde es interpretada como un mapa de vectores por cada pixel, los vectores tridimensionales son descritos por sus 3 coordenadas $\mathrm{X} 1, \mathrm{X} 2$ y $\mathrm{X} 3$, cuyos valores son asignados por los valores RGB de cada pixel de esta manera: $X 1=R, X 2=G$ y X3 = B.

El motor gráfico toma el objeto 3D, luego aplica el mapa de normales sobre el objeto de la misma manera que lo hace con la textura, el motor gráfico interpretará esto como un relieve utilizando las luces y el ángulo de la cámara. Esta técnica permite conseguir más nivel de detalle sacrificando menos recursos de máquina en tiempo real.

Para crear un mapa de normales se debe utilizar una herramienta 3D que tenga estas capacidades como las últimas versiones de Maya o de 3DMax que pueda plasmar el relieve de un modelo de un alto número de polígonos en una imagen $2 \mathrm{~d}$ y luego poder aplicarlo a un modelo $3 \mathrm{D}$ de bajo nivel de polígonos y que el resultado visual en el motor gráfico sea casi idéntico al modelo original. En este punto se siguieron las siguientes etapas:

Crear modelo de alto nivel de detalle en polígonos: Lo primero que se debe tener es un modelo detallado lo cual se logra con un número elevado de polígonos.

Generar mapa de normales: Teniendo el modelo complejo se selecciona la cámara que sería el punto de referencia de cada vector en la superficie, en este caso, es una cámara ubicada en el tope de la escena y cuyo foco es la superficie; para ello se utilizó un motor de render de Maya llamado Mental Ray.

Aplicación del mapa de normales: El mapa generado es una imagen que contiene información de la superficie con respecto al observador, para que los motores gráficos puedan interpretar el mapa normal correctamente se debe asignar en las propiedades de relieve o bump en inglés, una vez asignado el resultado es bueno pero lo más relevante es la reducción de polígonos de 486490 a 1 , lo que permite tener modelos más detallados visualmente y de geometría más sencilla facilitando la ejecución en tiempo real.

\section{Proceso de Texturización y Baking}

Estos dos procesos ajenos de la realización del modelo, son procesos que necesitaron más tiempo de trabajo, el primero porque necesita de un detallado y meticuloso trabajo en el cual, las texturas deben ser procesadas para darle continuidad entre los diferentes objetos. En la Fig. 5a, se puede ver sin renderizar un sector de un Bloque en el que la zona resaltado con verde, demarca un objeto diferente de los otros sectores del bloque; el trabajo de continuidad de texturas consiste en hacer que entre objetos concuerde tamaño y forma de la textura como sería el caso de la textura de ladrillo, esto para brindar mayor realismo. En la Fig. 5b, se ve como entre línea divisoras se le nota la continuidad de la textura entre plano y plano, de esto se trata este proceso, lograr esto en cada punto de convergencia de los bordes de los planos de un objeto, y después a mayor escala, la continuidad en puntos de convergencia de bordes de diferente objetos, en este caso entre piso y piso de cada bloque.

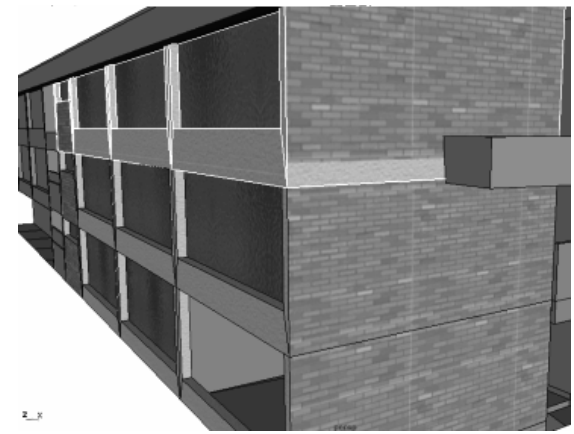

a.

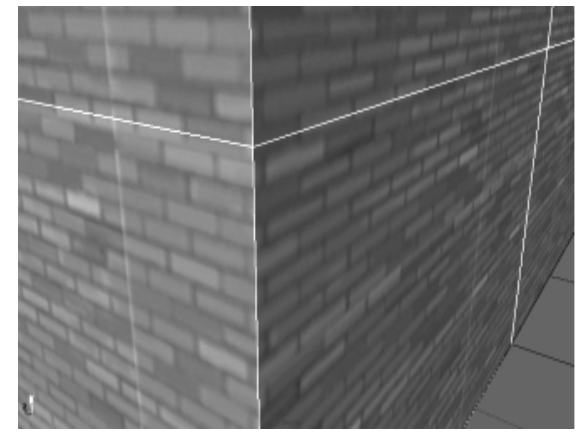

b.

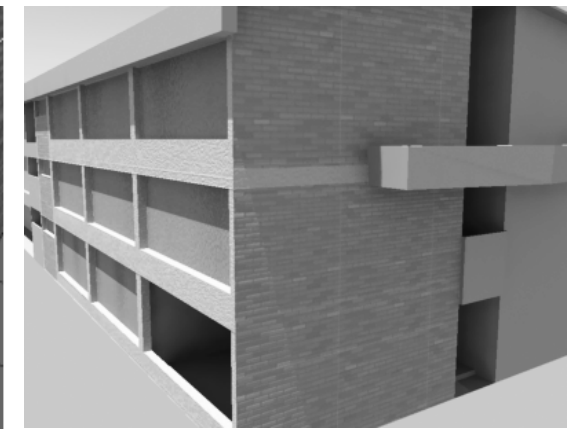

C.

Fig. 5. a. Texturizado en un Bloque. b. Continuidad entre planos. c. Baking del bloque

Otro de los procesos es el Baking, que es un proceso donde el software genera archivos de imagen de un objeto totalmente renderizado, es decir, el software utiliza la textura del objeto, 
sobre esta calcula las luces, sombras y otros tipos de elementos que afectan su acabado final y lo guarda en un archivo de imagen, esto con el fin de que posteriormente el mismo software $u$ otro como un motor gráfico puedan evitar el proceso de renderizado con software y muestren el acabado final del objeto en tiempo real (Ver Figura 5c).

En la Fig. 5c, se observa una imagen en las que las sombras y demás están calculadas con respecto a la luz. Este proceso es más dependiente de la máquina con que se hace, ya que un Bake de un objeto puede durar mucho tiempo, dependiendo de las sombras y la luz que ese objeto reciba, y alternamente la capacidad de procesamiento de la máquina en que se realice. En la Fig. 6, se observa un mosaico de varias zonas de la Institución.

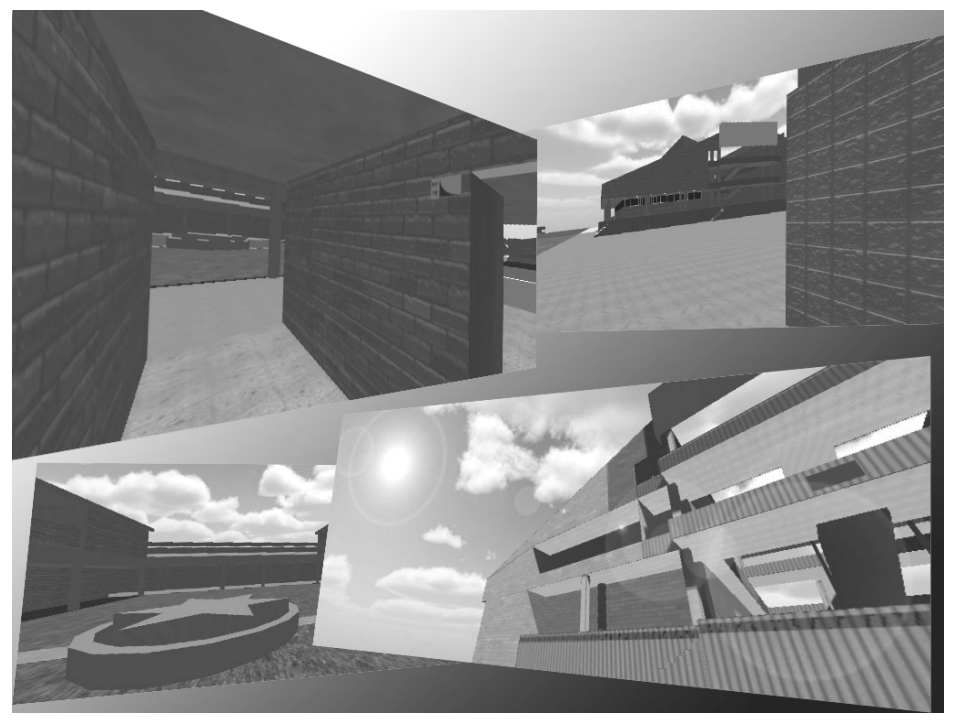

Fig. 6. Entorno Virtual del Politécnico

\section{CONCLUSIONES}

En este trabajo se planteó el desarrollo de un Entorno Virtual del Politécnico Colombiano Jaime Isaza Cadavid, con Realidad Virtual. En el mencionado desarrollo, se utilizó Autodesk entre varias herramientas de modelado 3D y Unity como motor gráfico; posteriormente, se hace una amplia explicación de la creación de mapas UV con sus procesos de limpieza de geometría, unión de vértices y la creación de los mapas basado en dirección de las rectas normales con respecto al objeto y sus caras; en el proceso de texturización, renderizado y baking se tuvo en cuenta la simulación de los procesos físicos de la naturaleza, como lo es, la emisión de luz y la refracción de los fotones contra los planos.

Como trabajo futuro se plantea tratar el problema de la refracción de los fotones y la incorporación de un personaje que guíe el entorno virtual.

\section{REFERENCIAS}

Bastanlar, Y., Grammalidis, N., Zabulis, X., Yilmaz, E., Yardimcia, Y., Triantafyllidis, G. 3D reconstruction for a cultural heritage virtual tour system. the International Archives of the Photogrammetry, Remote Sensing and Spatial Information Sciences. Vol. XXXVII. Part B5. Beijing (2008).

Bellotti, F., Berta, R., Cardona, R. DeGloria, A. An architectural approach to efficient 3D urban modeling. Computers \& Graphics 35 1001-1012 (2011).

Carpenter, T., Doerfler, G., Way, T. y Klassner, F. An Approach to Maintaining Viewer Perspective in Interactive Virtual Tours. The 2011 International Conference on Computer Graphics and Virtual Reality (CVGR 2011), Las Vegas, Nevada, July 18-21 (2011). 
Mármol P., Villao A. y F. Avilés. Aplicación Multimedia para Construir un Recorrido Virtual por la Torre del Reloj. Escuela de Diseño Gráfico y Comunicación Visual (2008).

Matteo A., Coto E., Navarro H. y O. Rodríguez. Una herramienta para generar Mundos Virtuales Inmersivos. Revista Lectura en Ciencias de la Computación, SIN 1316-6239 (2001).

Monedero, J. Recorrido interactivo por escenarios virtuales de grandes dimensiones. SIGraDi, bibio (2001).

Quintero, C. Sierra E., y W. Sarmiento. Diseño de un prototipo de sistema de realidad virtual inmersivo simplificado. Revista Ciencia e Ingeniería Neogranadina., Volumen 18, Número 001, Pág. 35-50. Junio (2008).

Ramírez, I., Córdoba G. y Rodríguez R. Diseño e implementación del módulo motivacional del proyecto Yatiqasiña. Revista Facultad de Ingenierías, UTA, Volumen 5 - Chile (1998).

Rodríguez A. Prototipo tridimensional para simular un recorrido virtual por las instalaciones de la Universidad El Bosque. Trabajo de grado para optar el título de Ingeniero de Sistemas (2007).

Shukol, A. Young, K., Rushforth, E. 3D Modeling of Indoor Surfaces with Occlusion and Clutter. Proceedings of the 2011 IEEE International Conference on Mechatronics April 13-15,Istanbul, Turkey (2011).

Sun, K., Chan, H., Yin, T. A Case Study on Building Web3D Virtual Reality and Its Applications to Joyful Learning. Digital Content, Multimedia Technology and its Applications (IDCTA), 2011 7th International Conference on 12 September (2011).

Valencia, M. y D. Vargas. Análisis, Diseño e Implementación de un software para visitas virtuales $3 D$ interactivas por instalaciones de la Universidad Tecnológica de Pereira. Trabajo de grado para optar el título de Ingeniero de Sistemas (2007).

Wang, Y., Mei, L., Li, Q., Wang, J., Zhu, Z. 3D Virtual Client Center and Its Service Oriented Modeling. 2011 International Joint Conference on Service Sciences. IEEE Computer Society. (2011).

Wang, C. y Wu, S. An Adaptive RFID Localization Mechanism Supporting 3D Virtual Tour System. Ubi-Media Computing, 2008 First IEEE International Conference on July 31 2008-Aug. 1 (2008).

Xu, H., Zhuang, H., Hou, X., y Ge, J. An Intelligent Project Agency for Web-3D Virtual Trading Community Based on Google Earth. Computer Science and Information Technology, 2009. ICCSIT 2009. 2nd IEEE International Conference on Issue Date: 8-11 Aug (2009). 